Proyecciones Journal of Mathematics

Vol. 28, No 3, pp. 227-232, December 2009.

Universidad Católica del Norte

Antofagasta - Chile

\title{
ALGEBRAIC PROPERTIES AND EXAMPLES OF INVERSE SEMIGROUPS
}

\author{
K. V.R. SRINIVAS \\ and \\ R. NANDAKUMAR \\ REGENCY INSTITUTE OF TECHNOLOGY, INDIA \\ Received : November 2008. Accepted : July 2009
}

\begin{abstract}
In this paper mainly we have obtained some important algebric properties of inverse semigroups.
\end{abstract}

Key words : Retraction, Inverse semigroup.

AMS subject classification : $20 M 18$. 


\section{Introduction :}

In this paper we have obtained some important properties of inverse semigroups. In theorem 1.1 it is observed that any inverse semigroup is an equationally defineable algebra and any homomorphic image of an inverse semigroup is again an inverse semigroup, (which is actually the theorem 7.36 of Clifford and Preston, vol-II). Any subalgebra of an inverse semigroup is again an inverse semigroup and the direct product of any family of an inverse semigroups is an inverse semigroup. Further if $S$ is an inverse semigroup, then a number of equivalent condition for $x^{-1} y$ to be an idempotent of $S(x, y \in S)$ are obtained(see theorem 1.3). It is also observed that if for any $a, b \in S$ with $a \wedge b$ exists and is equal to $(a \wedge b)^{-1}$. It is interesting to observe that if $a^{-1} b$ is an idempotent of $S$, then for any $c \in S, a c \wedge b c$ exists and is equal to $(a \wedge b) c$ and $c a \wedge c b$ also exists and is equal to $c(a \wedge b)$ which are obtained in theorems1.4 and 1.5. Further a number of equivalent conditions for any two elements a and a- 1 of inverse semigroups to commute is obtained in theorem 1.6. Finally in theorem 1.7 it is obtained that, if $S$ is an inverse semigroup in which $a$ and $a^{-1}$ commute $\forall a \in S$, then every congruence relation on $E(S)$ can be extended to a congruence on $S$.

First we start with the following preliminaries:

Def:0.1 : An element a of a semigroup $S$ is said to be regular if there exists $x \in S$ such that axa=a. A semigroup $\mathrm{S}$ is called regular if every element of $S$ is regular.

Def:0.2 : Two elements $a$ and $b$ of a semigroup S are said to be inverse of each other iff $a b a=a$ and $b a b=b$.

Def:0.3 : By an inverse semigroup we mean a semigroup in which every element has a unique inverse.

Def:0.4 : Let A be a universal algebra and B be a subalgebra of $A$ then $B$ is called a retract of $\mathrm{A}$ if there exists a homomorphism $f: A \rightarrow B$ such that $f$ is restricted to $B$ is identity on $\mathrm{B}$. B is called retract and $\mathrm{f}$ is called a retraction mapping.

Def:0.5 : Let $S$ be a semigroup and let $a \in S, L(a)$ denotes the principal left Ideal $S^{\circ} a$ and R(a) denotes the principal right Ideal $a S^{\circ} ; L$ represents the relation $(a, b) \in S \cdot S: L(a)=L(b) R$ representes the relation $(a, b) \in S \cdot S: R(a)=R(b)$. $D$ representes the relation $L \circ R$.

Theorem 1.1 : A system $(S, \cdot,-1)$ is an inverse semigroup iff

a) $(x y) z=x(y z) \forall x, y, z \in S$

b) $(x-1)^{-1}=x$.

c) $\left(x x^{-1}\right)\left(y y^{-1}\right)=\left(y y^{-1}\right)\left(x x^{-1}\right)$ 
d) $x x^{-1} x=x$;

Proof : It is obvious.

Remark 1.1 : Since an inverse semigroup is equotionally definable from the general theory of W-algebras satisfying identity (a),(b),(c),(d). From the above we have the following corollaries

Corollary 1.1 : An homomorphic image of an inverse semigroup is again an inverse semigroup (which is actually the theorem 7.36 of Clifford and Preston,vol-II).

Corollary 1.2 : Subalgebra of an inverse semigroup is again an inverse semigroup.

Corollary 1.3 : Direct product of any non-empty family of inverse semigroups is again an inverse semigroup.

The following examples establish the independency of the axioms mentioned in the above theorem.

Example 1.1 : Let $S=e, a, b$ Define '-1' in $\mathrm{S}$ by the compostion table as follows and define ${ }^{\prime}-1^{\prime}$ by $a-1=a, b-1=b$ and $e-1=e$.

\begin{tabular}{|llll|}
\hline$\cdot$ & $e$ & $a$ & $b$ \\
$e$ & $e$ & $a$ & $b$ \\
$a$ & $a$ & $e$ & $a$ \\
$b$ & $b$ & $a$ & $e$ \\
\hline
\end{tabular}

Then $(S, \cdot,-1)$ is a system satisfying (b),(c),(d) but not (a). Thus (a) is not a consequence of the remaining.

Example 1.2 : Let $S=e, a, b$ Define ' -1 ' in $\mathrm{S}$ by the compostion table as follows and define ' $-1^{\prime}$ in $S$ by $a-1=b \forall a \in S$

\begin{tabular}{|llll|}
\hline & $e$ & $a$ & $b$ \\
$e$ & $e$ & $e$ & $e$ \\
$a$ & $e$ & $a$ & $b$ \\
$b$ & $e$ & $a$ & $b$ \\
\hline
\end{tabular}


Then $(S, \cdot,-1)$ is a system satisfying (a),(c),(d) but not (b). Thus (b) is not a consequence of the remaining.

Example 1.3 : Let ' $X^{\prime}$ be a set with at least two elements. Take $S=X \times X$ and define '-1' in $\mathrm{S}$ by $\left(x_{1}, y_{1}\right)\left(x_{2}, y_{2}\right)=\left(x_{1}, y_{2}\right)$ and define '-1' by $\left(x_{1}, y_{1}\right)-$ $1=\left(y_{1}, x_{1}\right)$. Then $(S, \cdot,-1)$ is a system satisfying (a),(b),(d) but not (c). Choose $x_{1}, x_{2} \in X$ with $x_{1} \neq x_{2}$ and let $y_{1}, y_{2} \in X$, Then $\left(x_{1}, y_{1}\right)\left(x_{1}, y_{1}\right)-$ $1\left(x_{2}, y_{2}\right)\left(x_{2}, y_{2}\right)^{-1}=\left(x_{1}, x_{2}\right)$ and $\left(x_{2}, y_{2}\right)\left(x_{2}, y_{2}\right)^{-1}\left(x_{1}, y_{1}\right)\left(x_{1}, y_{1}\right)^{-1}$ $=\left(x_{2}, x_{1}\right) \neq\left(x_{1}, x_{2}\right)$. Thus (c) is not a consequence of the remaining.

Example 1.4 : Let $(S, \cdot)$ be a semigroup of positive integers under ordinary multiplication. Define ${ }^{\prime}-1^{\prime}$ in $S \times S$ by $(a, b)^{-1} \cdot(c, d)=(a c, b d) \forall a, b, c, d \in$ $S$. Define '-1' in $S \times S$ by $(a, b)^{-1}=(b, a)$. Then $S$ satisfies (a),(b) and (c) but not $(\mathrm{d})$, as $(2,1)(2,1)-1(2,1)=(2,1)(1,2)(2,1)=(4,2) \neq(2,1)$.

The following theorem is already proved in Clifford and Preston vol-II: But here an alternative proof is given for the part $a \leq b \Longrightarrow a c \leq b c \forall c$.

Theorem 1.2 : Let $S$ be an inverse semigroup. Let $a, b \in S$. If $a \leq b$ then $a c \leq b c$ for any element $c$ of $S$.

Proof : Assume $a \leq b$ and let $c \in S$. Then we have $a-1 \leq b-1$ and therefore $c-1 a-1 \leq c-1 b-1$ and So that $\left(c^{-} 1 a^{-} 1\right)^{-1} \leq\left(c^{-} 1 b^{-} 1\right)^{-1}$ and hence $a c \leq b c$.

In the following theorem a number of equivalent conditions on the elements $x$ and $y$ of an inverse semigroup are obtained in order to $x^{-} 1 y$ is an Idempotent of $S$.

Theorem 1.3 : If $S$ is an inverse semigroup and $x, y \in S$, then the following are equivalent

1) $x^{-1} y$ is an Idempotent of $S$

2) $y^{-1} x$ is an Idempotent of $S$

3) $y y-1 x \leq y$ in the natural partial order on $S$

4) $x x-1 y \leq x$ in the natural partial order on $S$

5) G. L. B of $x$ and $y$ exists and is equal to $x x^{-1} y$

6) G. L. B of $x$ and $y$ exists and is equal to $y y^{-1} x$

7) G. L. B of $x^{-1}$ and $y-1$ exists and is equal to $y^{-} 1 x x^{-1}$

8)G.L.Bof $\mathrm{X}^{-1}$ and $y-1$ exists and is equal to $x^{-1} y y^{-1}$

9) $\mathrm{x}^{-1}$ yy ${ }^{-1} \leq y-1$

10) $y^{-1} x x^{-1} \leq x-1$ 
The following example shows that there is an inverse semigroup in which $\mathrm{f}, \mathrm{g}$ have g.l.b and $\mathrm{f}-\mathrm{g}$ is not an Idempotent.

Let $X=1,2,3,4$ be a finite set. Let $f: 1,2,3 \rightarrow 2,3,4$ defined by $f(1)=2 ; f(2)=4 ; f(3)=3 ; g: 1,3,4 \rightarrow 2,4,1$ is defined by $g(1)=$ $2 ; g(4)=3 ; g(3)=1$. Domain of $f^{-1} g=(1,2,3, \cap 1,3,4) f=1,3 f=2,3$. But $(3)\left(\mathrm{f}^{-1} g\right)=(3) g=1$; thusf ${ }^{-1} g$ is not an Idempotent in the domain of $f^{-1} g$.

Also define $h: 1 \grave{a} 2$ by $h(1)=2$. then clearly $f$ and $g$ are non-comparable and $h \leq f, h \leq g$ and hence $h$ is a lower bound of $f$ and $g$ and it is greatest lower bound, thus in an inverse semigroup G.L.B of $f$ and g may exist even though $f^{-1} g$ is not an idempotent of $S$.

Theorem 1.4 : Let $\mathrm{S}$ be an inverse semigroup and let $a^{-1} b$ be an idempotent element of $S$, for $a, b \in S$. Then for any $c \in S, a c \wedge b c$ exists and is equal to $(a \wedge b) c$.

Proof : Proof is clear

Theorem 1.5 : Let $a, b \in S$ and let $a^{-1} b$ be an idempotent of $S$, then for any $c \in S, c a \wedge c b$ exists and is equal to $c(a \wedge b)$.

Proof : Proof is clear.

In the following theorem a number of equivalent conditions on an inverse semigroup $S$ are obtained in order to the elements $a$ and $a^{-1}$ commute $\forall a \in S$.

Theorem 1.6 : In an inverse semigroup $S$ the following are equivalent; $a$ and $a^{-1}$ commute $\forall a \in S$, equivalently $\mathrm{S}$ in a Clifford semigroup $e a=a e \forall$ idempotents $e$ and $\forall a \in S$. la ra- 1 is an endomorphism of $\mathrm{S}$, where la is the inner left translation and $r a^{-1}$ is the inner right translation. The mapping $a \rightarrow a a^{-1}$ is a retraction of $S$ onto $E(S)$. The mapping $a \rightarrow a a^{-1}$ is inverse preserving. $\mathrm{L}$ is a congruence relation. $L=R . L \subseteq R . R \subseteq L L=R=D$. $S$ is a set union of groups.

Theorem 1.7 : If $S$ is an inverse semigroup in which $a$ and $a^{-1}$ commute $\forall a \in S$, then every congruence relation on $\mathrm{E}(\mathrm{S})$ can be extended to a congruence on $S$.

Proof : Since $E(S)$ is a retract of $\mathrm{S}$ and $\mathrm{S}$ is an universal algebra, the theorem follows. 


\title{
References
}

[1] A. H. Clifford and G. B. Preston, The algebric theory of semigroups, American Mathematical Society, Providence, Vol-1, (1964).

[2] A. H. Clifford and G. B. Preston, The algebric theory of semigroups, American Mathematical Society, Providence, Vol-2, (1967).

\author{
K. V. R. Srinivas \\ Regency Institute of Technology, \\ Yanam-533464 \\ India \\ e-mail: srinivas_kandarpa06@yahoo.co.in \\ and
}

\section{R. Nandakumar}

Regency Institute of Technology, Yanam-533464

India

e-mail: rnandakumar2001@yahoo.com 